Revue des sciences de l'éducation

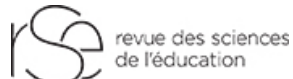

\title{
Des sentiments aux connaissances littéraires : travail de transaction des mots, construction des valeurs au primaire et analyse de corpus \\ Sentiments related to literary knowledge : transaction of words and values construction at elementary school and corpus analyses \\ De los sentimientos a los conocimientos literarios : trabajo de “transacción” de palabras, construcción de valores en la primaria y análisis de corpus
}

Martine Champagne-Vergez

\section{Volume 39, numéro 1, 2013}

La littérature de jeunesse : le lecteur, l'oeuvre, les passeurs et le passage

Texte reçu le : 5 juin 2011, version finale reçue le : 17 juillet 2013, accepté le : 15 août 2013

URI : https://id.erudit.org/iderudit/1024533ar

DOI : https://doi.org/10.7202/1024533ar

Aller au sommaire du numéro

Éditeur(s)

Revue des sciences de l'éducation

ISSN

0318-479X (imprimé)

1705-0065 (numérique)

Découvrir la revue

Citer cet article

Champagne-Vergez, M. (2013). Des sentiments aux connaissances littéraires : travail de transaction des mots, construction des valeurs au primaire et analyse de corpus. Revue des sciences de l'éducation, 39(1), 61-89. https://doi.org/10.7202/1024533ar

\section{Résumé de l'article}

L'enseignant, passeur privilégié d'oeuvres littéraires, initie, à leur propos, une médiation par le biais du travail des mots, notamment d'ordre esthétique et émotionnel. Comment l'expression de l'émotion esthétique participe-t-elle de ce qui s'apprend durant les activités littéraires scolaires? Quelle est la relation entre les sentiments et la construction des valeurs? Notre étude repose sur trois corpus oraux et écrits, recueillis en fin d'école primaire en France $\left(5^{\mathrm{e}}\right.$ année). L'observation des actions langagières se situe à l'endroit des reprises discursives du débat interprétatif. Dans certains cas, des transactions, co-élaborées par les élèves et l'enseignant, à l'oral comme à l'écrit, portent la trace de cette élaboration des savoirs et des valeurs. 


\title{
Des sentiments aux connaissances littéraires: travail de transaction des mots, construction des valeurs au primaire et analyse de corpus
}

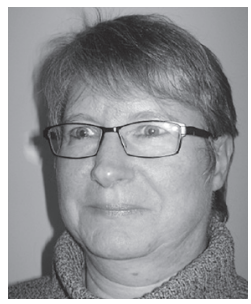

Martine Champagne-Vergez

Maître de conférences

Université de Bordeaux 4

\begin{abstract}
RÉSUMÉ • L'enseignant, passeur privilégié d'œuvres littéraires, initie, à leur propos, une médiation par le biais du travail des mots, notamment d'ordre esthétique et émotionnel. Comment l'expression de l'émotion esthétique participe-t-elle de ce qui s'apprend durant les activités littéraires scolaires? Quelle est la relation entre les sentiments et la construction des valeurs? Notre étude repose sur trois corpus oraux et écrits, recueillis en fin d'école primaire en France ( $5^{\mathrm{e}}$ année). L'observation des actions langagières se situe à l'endroit des reprises discursives du débat interprétatif. Dans certains cas, des transactions, co-élaborées par les élèves et l'enseignant, à l'oral comme à l'écrit, portent la trace de cette élaboration des savoirs et des valeurs.
\end{abstract}

MOTS CLÉS - actions langagières, activités littéraires scolaires, débat interprétatif, élaboration des savoirs, valeurs.

\section{Introduction et problématique}

Dans le cadre de cet article, nous allons traiter de questions didactiques qui interrogent la transmission des savoirs en littérature et qui peuvent être liées à la construction sociale d'un sujet, lecteur d'œuvres littéraires, et cela, dans le but de mieux comprendre ce sujet littéraire scolaire. Le sujet lecteur, complexe et objet de multiples descriptions, renvoie à la tradition ou à l'ordre social, en vue de la construction de sens, diversement finalisé selon les auteurs. À l'école, on n'attend pas du lecteur scolaire une lecture globale du texte ni un plaisir éprouvé relaté (Daunay, 2007). Entre les jeux de lecture, la littérarité (comme caractérisation du littéraire, liée aux dimensions fictionnelle et linguistique des textes, ainsi qu'à la sollicitation de l'expérience du lecteur), l'intertextualité (lien entre les textes), voire l'architextualité (relation entre les différentes sources des textes et leurs 
différentes formes), les instances lectoriales, les distanciations et les allers-retours (Butlen et Dubois-Marcoin, 2005), cet article vise la dimension pragmatique d'une analyse de pratiques effectives. Le lecteur scolaire est à la fois singulier, acteur et sujet, force critique, entre subjectivation et rationalisation, par la négociation et la médiation des œuvres littéraires (Boiron, 2008). Comment cette expérience par procuration se manifeste-t-elle à l'école par l'action collective, en particulier à propos de la construction du personnage, sollicité depuis le début de l'école primaire?

L'objet de cet article concerne la problématique suivante: comment l'école institue-t-elle l'émergence d'un sujet singulier, lecteur-interprète, pour construire des valeurs esthétiques, morales, qui contribuent à la construction de connaissances littéraires et humanistes, à partir de l'énonciation d'un point de vue plus ou moins subjectif? Quel rôle remplit la mise en mots des sentiments et quelle construction des valeurs est envisageable en fin d'école primaire au sein du débat de lecture littéraire; en particulier quant à la construction de l'identité référentielle des personnages (Reuter, 1998; Tauveron, 2002)? Par ce biais, quelles modalités pédagogiques ou didactiques faciliteraient les médiations enseignantes et le rôle de l'enseignant en tant que passeur culturel?

Notre étude se focalise sur le conflit potentiel lié aux tensions qui donnent lieu à des manifestations de ruptures, d'incohérences apparentes, ici nommées hiatus (Champagne-Vergez, 2010a). Ces contradictions sont l'objet de considérations méthodologiques d'identification des hiatus et de leur devenir. L'hypothétique participation des élèves à la construction du problème, en termes de contenus de savoir et de la résolution potentielle du problème, font du conflit un point d'observation pour analyser des productions cognitivo-langagières liées à la construction des significations.

Un premier temps sera consacré aux entrées théoriques concernant la compréhension et l'interprétation des œuvres.

Dans un deuxième temps, méthodologique, nous étudierons des niveaux d'échange à caractériser entre les interlocuteurs pour identifier des contenus d'apprentissage en cours d'élaboration. Des actions langagières (ChampagneVergez, 2010a) sont à la fois relatives au mécanisme général de l'activité d'un groupe donné, à la connaissance du locuteur, à son interaction avec la production discursive, en tant que processus cognitivo-verbaux en cours. Ces actions permettent de suivre des contenus notionnels sollicités par le biais de trois corpus d'activités littéraires. L'étude est centrée sur l'expression des émotions esthétiques et des valeurs, à l'aide de l'exploitation d'outils caractérisant les discours scolaires.

Cette recherche, exploratoire, se propose d'étudier des dires en relation à la technique sociale des sentiments (Nonnon, 2008), en tant que médiation culturelle possible à l'école primaire. La sollicitation des émotions esthétiques en relation avec la construction des valeurs constitue le terreau d'un problème, dévolu aux élèves. 
Un dernier temps permettra de revenir sur les contenus étudiés et pondérera les conclusions à tirer quant à l'émergence de contenus d'enseignements littéraires (et culturels).

D’une manière générale, comme on le sait, le contenu des savoirs et les modalités de leur réalisation sont l'objet d'interrogations partagées, au sein de recherches didactiques et pédagogiques (Dubois-Marcoin et Tauveron, 2008, p. 3-26). Des pratiques pédagogiques à propos de la littérature de jeunesse infléchissent en France, comme dans les pays francophones, les activités littéraires à l'école primaire vers des pratiques plus innovantes. Depuis 2002, date de l'entrée de la littérature dans les programmes de l'école primaire française, celle-ci est progressivement devenue incontournable en tant que discipline faisant partie des sept piliers du socle commun de connaissances et de compétences de 2006 (Annexe 1). Selon Lelièvre (2009), la littérature relève du cinquième pilier de la culture humaniste (ou compétence générique), permettant l'accès à une culture patrimoniale et à une culture littéraire contemporaine. Ainsi, la culture humaniste contribue à la formation du goût et de la sensibilité. En parallèle, le développement d'une maîtrise langagière prend appui sur le premier pilier du socle de compétences : la connaissance de la langue. Ce pilier, centré sur l'apprentissage de la lecture et de l'écriture, est considéré essentiellement comme une maîtrise des outils de la langue (vocabulaire, orthographe, grammaire). Or, le domaine de la culture humaniste n'est pas à dissocier de la maîtrise de la langue en dehors des pratiques langagières (Bishop et Cadet, 2011) ni des sous-disciplines du français.

La littérature à l'école permet une ouverture aux œuvres, aux idées, une construction de références sur le monde. Elle interroge les rapports aux savoirs élaborés à l'école, les savoirs notionnels d'une littérature scolaire ne se construisant pas en dehors de sujets lecteurs (Langlade et Rouxel, 2004). Les actions langagières (Champagne-Vergez, 2010a) participeraient de la construction de précurseurs des valeurs morales ou à ses ébauches, au sein des échanges sociaux. Aussi la prise en compte des discours des élèves sera-t-elle centrale dans cet article. L'étude ou l'examen du travail des mots nous informe quant à la mobilisation des rapports aux savoirs langagiers, particulièrement lorsque des émotions esthétiques (Bakhtine, 1984) sont convoquées. Que peut-on dire du travail des valeurs engagé dès l'école primaire? Le langage, dont on fait peu état des modalités qu'il peut emprunter sur le terrain, semble transparent. Cette recherche participe à l'élaboration de modes d'intelligibilité des réalités scolaires évoqués par Crahay (2010). La démarche exploratoire éprouve la viabilité d'outils, afin de tester leur pertinence pour apprécier l'apprentissage effectué et les notions sollicitées afin de les rendre lisibles.

La caractérisation des registres langagiers étudiés rend compte d'une langue aux normes plurielles, de tonalité socioaffective, dépendante d'un contrat didactique. Ces oraux sont polygérés. Cependant, ces caractéristiques, similaires au départ, renvoient à des productions cognitivo-langagières aux variations importantes selon 
les conceptions des enseignants, et selon les usages en vigueur dans les classes. Une étude comparative s'impose.

\section{Contexte théorique}

Le champ théorique est composé de plusieurs entrées intriquées, liées à la construction de la notion de personnage en littérature, pour lequel les valeurs morales que celui-ci pourrait véhiculer ou l'émotion esthétique suscitée sont de réels enjeux d'apprentissage. Le premier point, la question des valeurs morales a une incidence sur certains des gestes professionnels des enseignants: Bucheton (2011) parle de logique d'arrière-plan lors des débats littéraires. Ainsi, le rapport éthique au langage, tout en étant différent pour le sujet élève et pour l'enseignant, fonde une communauté discursive restreinte (Charaudeau et Mainguenau, 2002, p. 106-108) à la classe. Le deuxième point, l'évocation de la technique sociale des sentiments (Vygotski, 2005) est à préciser dans le contexte scolaire. Même s'il est nécessaire que des émotions soient formulées par le biais du langage pour faire état de sentiments (Damasio, 2003), ce n'est pas une condition suffisante pour délimiter des contenus disciplinaires (par exemple, Tauveron, 2002).

Les usages que nous faisons des termes valeurs, débat, gestes, communauté, renvoient à des sphères de référence hors de leur champ d'application d'origine et présentent des variations. Pour ces raisons, la dimension heuristique de notion (Barré de Miniac, 2008) pour parler de ces termes est plus explicite.

\subsection{Valeurs morales}

Les valeurs morales peuvent être sollicitées selon deux aspects:

- les valeurs morales d'un personnage, plus ou moins accessibles au lecteurinterprète et que ce dernier s'approprie par identification partielle, par le biais d'un travail langagier au contact des œuvres littéraires. Ces valeurs morales se concrétisent aussi par l'accès à la parole, concernant de ce fait et l'élève et le maitre. La relation à la parole occupe un statut particulier: elle institue l'élève en tant qu'individu en devenir, futur responsable de ses dires. Pour ce faire, l'enseignant agit en médiateur, ce dernier facilitant l'expression des dires (Jorro, 1999). Avant d'être responsable de ses actes (sens premier de la valeur morale), et précisément par l'action de dire, le jeune sujet scolaire construit progressivement la valeur morale de ses actes (différente des normes à respecter), notamment par le langage;

- les valeurs morales, au sens éthique de l'élaboration progressive d'un jugement et d'un esprit critiques (Dumortier, 2001), font l'objet d'un apprentissage implicite, à expliciter, selon nous, en nous appuyant sur les échanges cognitivo-langagiers à l'école. La littérature participerait ainsi à la représentation des valeurs humaines, et ce, pour partie, avant leur éventuelle mise à l'épreuve dans la vie. Ainsi les valeurs morales, dont le socle commun fait état (compétences morales et civiques), sont-elles à construire. Cela est-il possible à l'école par le biais de 
la littérature? D’une façon générale, Gardin (2008) atteste du langage comme mode de subjectivation vis-à-vis de soi-même et des autres. En ce qui concerne l'école, Leclaire-Halté (2006) doute de la compatibilité de la convocation d'effets esthétiques et des valeurs morales en littérature. Pourtant Lahire (1993) précise que les activités discursives permettent aux élèves de tester des expériences personnelles projetées dans l'imaginaire. L'auteur évoque une éthico-pratique dont on peut se demander si elle est observable.

À la suite de ces auteurs, les questions des valeurs morales à l'école nécessitent d'identifier les registres discursifs et cognitifs sollicités. Cette double entrée de la lecture, esthétique ou morale, nous amène à préciser le contexte de l'activité langagière (au sens de Bronckart, 1997) lors du débat interprétatif et en particulier des gestes professionnels (Bucheton, 2011) qui le pilotent.

\subsection{Les gestes d'enseignement au cours du débat interprétatif}

Le débat interprétatif, souvent évoqué lorsqu'il s'agit de parler d'une œuvre, est abordé par de nombreux auteurs (dont Burgos, 1996; Dias-Chiaruttini, 2011; Gion, 2003; Jorro et Crocé-Spinelli, 2010). À l'école primaire, en littérature, la prise de parole est décrite par Jorro et Crocé-Spinelli (2010) à partir des gestes éthiques de l'enseignant (l'autorisation à la prise de parole, des attitudes de retenue protégeant les lecteurs, des modes de reconnaissance valorisant la portée d'un propos littéraire). Ces attitudes permettent la création d'un espace de parole favorable à l'accueil, au développement de points de vue subjectifs sur le texte, à leur mise en partage (Jorro et Crocé-Spinelli, 2010, p. 133). Ces démarches sont-elles suffisantes pour accéder au travail interprétatif (malgré des retours sur les avancées ou les processus interprétatifs) et à la construction de savoirs?

Le débat interprétatif construit deux axes de connaissances fondamentales: la construction du récit et le positionnement du lecteur. D'une part, l'intrigue narrative, décrite par Ricœur (1984), participe à l'élaboration de savoirs scolaires à propos des œuvres littéraires, en particulier à propos des personnages. La compréhension peut ne rester que factuelle, et l'interprétation (Tauveron, 1999) sollicite des mises en relations ou des inférences entre les éléments du texte et une réorganisation personnelle de l'intrigue établies à partir de connaissances du monde et d'une plus ou moins grande connivence littéraire. Ainsi, les illusions référentielles du récit diffèrent de la réalité. De ce fait, le lecteur peut construire des sens singuliers aux événements du récit. Grâce au langage, le lecteur peut aussi rechercher des significations partagées de ce récit avec autrui. Aussi la compréhension et l'interprétation sont-elles liées, tout en empruntant des formes distinctes des temporalités du monde de l'action. En conséquence, la sémiotisation des actions du récit permet la reconfiguration des personnages interpellant le sujet, lecteur singulier. D’autre part, le travail de littérature à l'école, entre l'éventuelle réticence (brouillage volontaire de l'auteur) et résistance (double sens) des textes (Tauveron, 1999), prend en compte des lectures à différents niveaux, entre 
lecture psychoaffective, distanciée et critique (Dufays, 2004), en vue d'étayer des conduites lectoriales interprétatives.

Le débat interprétatif n'est pas un allant de soi... où la lecture se substituerait à la compréhension, où le langage serait transparent et où le point de vue du lecteur serait un point de départ sur lequel s'appuyer (Champagne-Vergez, 2010a, p. 111). Quand bien même la polysémie serait inhérente au texte littéraire, elle ne pourrait être le prétexte à tout laisser dire, et tout ce qui se dit n'est pas explicite. Le débat interprétatif, dont il n'est plus directement fait état dans les instructions officielles françaises de 2008, est implicitement contenu par l'élaboration, l'appropriation et la constitution d'un point de vue d'élève-lecteur au cours des débats à l'école.

Ensuite, ce débat est relatif: ce n'est pas une exégèse de l'œuvre, ce serait davantage un débat autour des lectures possibles de l'œuvre littéraire. L'hypothèse de conflits - à résoudre -, liés à la construction de la problématisation d'un contenu disciplinaire par accumulation d'incompréhensions, est recevable par le chercheur. La résolution de tels conflits solliciterait une technique sociale des sentiments pour apprendre à débattre, à comprendre et à interpréter.

Dans la classe de littérature, le travail des mots permettrait la construction de savoirs ainsi que des façons d'y parvenir par l'intermédiaire de gestes professionnels divers (Bernié et Goigoux, 2005). Ces gestes se différencient en gestes langagiers, de mise en relation de savoirs, d'ajustement, d'effacement (Bucheton, 2011) au sein de communautés scolaires.

\subsection{Communauté discursive}

Les notions précisées et les éléments de réponse évoqués rendent certains aspects saillants: les relations entre des valeurs morales et humaines, la pratique des gestes professionnels guidant le débat interprétatif à l'école primaire et les particularités d'une communauté discursive scolaire utilisant une technique sociale des sentiments privilégiée. Le débat interprétatif repose sur un choix d'activités en vue de porter publiquement le débat dans une communauté scolaire donnée. La notion de communauté discursive scolaire (Bernié, 2002; Bernié, Jaubert et Rebière, 2003) s'élabore à partir de pratiques sociales construites par le groupe de la classe, à travers les expériences accumulées des sujets, par l'intermédiaire des pratiques, ici lectoriales, variées. Des modalités d'échange s'installent dans ce collectif et donnent sens aux genres discursifs. La communauté discursive fonctionne progressivement, en relation avec les communautés discursives dites savantes (en relation avec la transposition didactique, si le principe d'une transposition partielle est acceptable: elles seraient représentées par les instances littéraires universitaires ou sociales reconnaissant la littérarité de l'œuvre). Les questions qui agitent ces deux sphères ont des points communs. Les discours de la communauté reflètent des pratiques de référence de ce milieu littéraire (Martinand, 2000). 


\subsection{Sensibilité esthétique}

Étudier la littérature à l'école sous-entend aussi développer une sensibilité esthétique (Bakhtine, 1984) portée par les choix de l'auteur, qui interpelle le lecteur singulier. La pluralité des façons d'agir-parler-penser va de pair avec la transformation des représentations lectoriales d'un sujet qui s'implique dans la communauté discursive, à la fois par son énonciation et par la co-construction de connaissances socialement travaillées par le langage. La sollicitation de la sensibilité esthétique s'incarne dans les émotions ressenties (des manifestations physiologiques à la suite d'un travail sur l'œuvre) par l'individu, et fait appel à l'écart entre le réel et l'art. Ces émotions, esthétiques, sont à l'origine d'un sentiment social, transformant de façon complexe les sentiments ressentis par leur mise en mots.

\subsection{Technique sociale des sentiments}

La technique sociale des sentiments (Vygotski, 2003) est une notion reprise par Nonnon (2008) à la suite d'un constat effectué dans les années 2000. Des didacticiens de la littérature tentent alors de réhabiliter un sujet affectif. Ainsi, Langlade et Rouxel (2004, parmi d'autres) veulent redonner une place à une lecture subjective facilitant des interprétations littéraires par l'implication du lecteur scolaire. Entre l'implication par motivation et l'implication dans la construction de l'objet d'enseignement, il y a un écart au centre de nos préoccupations.

$\mathrm{Au}$ sein de cet article, les échanges étudiés dans les classes renvoient aux échanges sociaux négociés au cours de transactions esthétiques (Rosenblatt, 1995, p. 217 : citée par Hébert, 2002 p. 120), faisant de la technique sociale des sentiments une entrée privilégiée du travail des mots en littérature.

Vygotski (1998) précise que l'expérience, l'émotion et la conscience des événements, tant physiologiques que psychologiques, ne sont pas à dissocier ou à hiérarchiser; et qu'il s'agit plutôt de comprendre leurs rapports internes par le biais de la recherche. Plus récemment, Damasio (2003), et d'une façon plus expérimentale le champ des neurosciences, confirment que l'émotion, physiologique, peut devenir consciente lorsqu'elle prend la forme de sentiments, ce que Vygotski (2003) envisageait déjà.

Ainsi, à l'école, le lecteur averti, si l'enseignant lui en laisse le temps et si on lui propose des situations reposant sur cette prise de conscience qui recouvrirait la valeur esthétique des mots, se met en mouvement. Des enjeux littéraires, s'ils sont accessibles, permettent aux élèves d'élaborer, d'anticiper des mondes à vivre, à travers des fictions littéraires. Cette projection scolaire se fonderait sur une construction progressive de valeurs (morales et humaines) par le biais des actes langagiers. Dans ce sens, l'acte langagier s'inscrit dans un fonctionnement normé des échanges constitutifs du statut de la personne, défini ainsi par Jacques:

Selon l'éthique, un individu vivant accède à la personne en tant qu'il s'accomplit dans un acte moral normé, effectué avec les autres et envers les autres. Selon nous, 
un des traits définissants de la personne (interlocuteur possible) est la compétence communicative et la capacité qu'elle implique à se poser en communiquant avec la parole (2007, p. 49).

Vygotski, à l'origine de la formulation de la technique sociale des sentiments (1998), précise que la conscientisation des émotions, par leur expression au moyen du langage en sentiments, entraîne des tensions relatives, chacun éprouvant des affects divers. Ces tensions entre des formes reconnues puis socialisées transposent ce qui est vécu physiologiquement dans un domaine plus conscientisé. La verbalisation des émotions ressenties, ou expression des sentiments, constitue un travail des mots dans un espace de conflits potentiels. Nonnon (2008) insiste sur ce conflit préalable au travail des mots, indispensable à la construction des significations inhérentes au langage qui devient objet d'apprentissage, comme nous allons en proposer un exemple dans la construction de l'identité des personnages et des relations qui les unissent (Tableau 1). Si des conflits cognitivo-langagiers sont potentiellement présents, la concrétisation des conflits liés aux valeurs esthétiques et morales, voire humaines, alimente la réflexion orale au sein de la communauté discursive scolaire. Cette concrétisation potentielle est porteuse de la construction d'un problème ou d'une problématisation littéraire.

Les notions de valeurs morales, gestes $d u$ débat littéraire et technique sociale des sentiments sont étudiées par Nonnon (2008), à partir de corpus recueillis au collège ou au lycée. Ces approches se retrouvent à l'école primaire, dans des limites que nous tenterons de préciser.

\subsection{Synthèse}

Dans les corpus cités dans cet article, le débat interprétatif n'est pas le lieu d'un consensus à établir ni de contenus définitifs, mais un espace de questionnement, enrichi de références, où les relations entre les textes font état d'expériences diverses et personnelles. Les expériences, rendues publiques, interrogent chaque membre de la communauté discursive de la classe. Ces dires publics sont à l'origine d'échanges, d'expériences à partager, et deviennent intersubjectifs. Des réflexions, des démarches de vérification des dires convoquent des mondes par procuration. De tels échanges sont relayés par des transactions (Rosenblatt, 1994: citée par Hébert, 2002, p. 148) constitutives d'une problématisation.

La communauté où se déroulent ces transactions est particulière. La complexité des positionnements énonciatifs attendus des élèves se superpose à leur intrication. L'analyse et l'orientation de ces reconfigurations sont peu possibles en temps réel pour l'enseignant. De ce fait, les actions sur le milieu didactique paraissent limitées. Suivre la manière dont les activités scolaires sont guidées au sein des interactions nourrit l'investigation.

Les gestes des enseignants n'expliquent pas comment des valeurs morales discursives participent à l'élaboration de valeurs, même lors de la dévolution de la situation. D’après Rosier (2010), la coélaboration du problème littéraire, en 
particulier, et sa concrétisation restent une difficulté des mises en œuvre didactiques et pédagogiques. Cette délégation d'autorité, ou lâcher-prise des enseignants, évoquée par les didacticiens de la littérature, pour le collège ou le lycée (Ahrs, 2007), englobe l'édification d'une communauté discursive littéraire. Cependant, les modalités d'existence de ces communautés discursives font que la reproductibilité méthodologique reste délicate. Les activités des communautés refléteraient des pratiques sociales de référence d'un milieu littéraire extra-scolaire. Or, à l'école primaire, ces activités sont définies en vue de savoirs à atteindre (Tauveron, 1999) qui divergent des pratiques sociales de référence (les communautés discursives savantes ou littéraires). Ce débat diffère aussi des variables de l'ingénierie didactique, où l'avancée du temps didactique ne relève pas seulement du milieu, mais aussi du contenu des transactions.

\subsection{Entre enseignement et apprentissage : objectifs spécifiques}

Étudier finement et analyser la façon dont s'élaborent les contenus notionnels et les processus selon lesquels ces apprentissages se disent, à travers le suivi des mouvements discursifs (François, 2005), relèvent de deux axes : les reprises-modifications et les stratégies énonciatives.

1) Les reprises-modifications

Les reprises sont moins le lieu d'une répétition ou d'une modification que d'un mouvement discursif (François, 1989) accompli à travers les discours des interlocuteurs. Les reprises thématiques, visibles jusqu'à 12 fois, pour les adieux d'un grand-père à son petit-fils (Tableau I), permettent de découper les corpus. Tout au long des échanges, ces reprises-modifications s'enchevêtrent. Ce constat, marquant, nous interroge et nous incite à étudier les écarts entre ces reprises.

\section{2) Les stratégies énonciatives}

À l'école, la langue est un outil social normé, reconnu et utilisé. Différentes stratégies énonciatives sont identifiables au sein des conventions énonciatives telles que Jacques (1988) les envisage, dans des situations différentes (Tableau 1). Ces situations (conversation, négociation, dialogue) sont caractérisées en niveaux de plus en plus denses, en relation avec un contenu discursif convoqué. La typologie, initiée par l'auteur et poursuivie (ajout de discussion), prend en compte la généralité, l'homogénéité, la convention, la relation, l'objectif, la fonction d'un propos au sein d'un continuum d'échanges.

Différents niveaux d'information et de sollicitation (la prise en compte des partenaires et le déroulement, les formes, les contenus, les finalités de l'échange) s'appuyant sur différents écrits de Jacques $(1979,1988)$ renseignent sur les discours singuliers des partenaires d'une communauté discursive.

La conversation (qui montre une sensibilité aux événements sociaux), la négociation (porteuse d'un conflit pratique et non dénuée de pouvoir) ou le dialogue 


\section{Tableau 1}

Essai d'identification des constructions de significations socio-langagières (stratégies énonciatives établies d'après Jacques [1985, 1988])

\begin{tabular}{|c|c|c|c|c|}
\hline & Conversation & Discussion & $\begin{array}{l}\text { Négociation } \\
\text { (négociation, } \\
\text { entretien) }\end{array}$ & $\begin{array}{l}\text { Dialogue } \\
\text { (débat, controverse, } \\
\text { confrontation) }\end{array}$ \\
\hline $\begin{array}{l}\text { Situation des } \\
\text { partenaires }\end{array}$ & $\begin{array}{l}\text { Places non allouées } \\
\text { Aspect cérémoniel } \\
\text { Contexte culturel } \\
\text { plutôt homogène }\end{array}$ & $\begin{array}{l}\text { Discours } \\
\text { dominant } \\
\text { Partenaires du } \\
\text { même domaine }\end{array}$ & $\begin{array}{l}\text { Places attribuées } \\
\text { Adversaires, forces } \\
\text { en présence } \\
\text { Intérêts opposés }\end{array}$ & $\begin{array}{l}\text { Partenaires, } \\
\text { réciprocité } \\
\text { Efforts conjugués }\end{array}$ \\
\hline Déroulement & $\begin{array}{l}\text { Pas de progression } \\
\text { Réplique } \\
\text { secondaire } \\
\text { Accorder le discours } \\
\text { à son statut } \\
\text { Réparer une offense } \\
\text { Aspect ludique }\end{array}$ & & $\begin{array}{l}\text { Protocole d'accord } \\
\text { Ajustements } \\
\text { nécessaires } \\
\text { consentis } \\
\text { Capture } \\
\text { d'information } \\
\text { Information } \\
\text { complémentaire ou } \\
\text { de concession }\end{array}$ & $\begin{array}{l}\text { Interaction finalisée } \\
\text { Initiatives partagées } \\
\text { Requête de } \\
\text { confirmation } \\
\text { Élucide les règles de } \\
\text { sa production } \\
\text { Demande } \\
\text { d'information }\end{array}$ \\
\hline Formes & $\begin{array}{l}\text { Répliques } \\
\text { inachevées } \\
\text { Chevauchement } \\
\text { Observance de la } \\
\text { convention } \\
\text { Enchaînement } \\
\text { faiblement contraint }\end{array}$ & $\begin{array}{l}\text { Références et } \\
\text { arguments }\end{array}$ & $\begin{array}{l}\text { Actes initiateurs et } \\
\text { actes réactifs }\end{array}$ & $\begin{array}{l}\text { Argumentation plus } \\
\text { contrainte } \\
\text { Interaction verbale } \\
\text { finalisée }\end{array}$ \\
\hline Contenus & $\begin{array}{l}\text { Appartenance à } \\
\text { une } \\
\text { communauté } \\
\text { Contraintes } \\
\text { thématiques } \\
\text { (évocation, règles } \\
\text { de convergence) } \\
\text { Sujet collectif } \\
\text { Présupposés admis } \\
\text { Inférences } \\
\text { impossibles } \\
\text { Sensibilité à la } \\
\text { conjecture }\end{array}$ & $\begin{array}{l}\text { Cohérence } \\
\text { thématique } \\
\text { Thèses non } \\
\text { abordées } \\
\text { Inférences mises } \\
\text { en marge }\end{array}$ & $\begin{array}{l}\text { Objet transactionnel } \\
\text { Règles, stratégies, } \\
\text { marge étroite } \\
\text { Portée pratique } \\
\text { stabilisatrice } \\
\text { Compromis, } \\
\text { appauvrissement } \\
\text { Dissimulation } \\
\text { partielle }\end{array}$ & $\begin{array}{l}\text { Objet à construire } \\
\text { Objectif unique } \\
\text { Thèses mises en } \\
\text { commun, références } \\
\text { Inférences rappelées, } \\
\text { contrôlées } \\
\text { Objets symboliques } \\
\text { contrôlés à des fins } \\
\text { d'explicitation }\end{array}$ \\
\hline Finalités & $\begin{array}{l}\text { Gagner ou perdre la } \\
\text { face } \\
\text { L'infraction peut } \\
\text { être réparée } \\
\text { Désir de } \\
\text { reconnaissance } \\
\text { Principe de plaisir } \\
\text { de la parole }\end{array}$ & $\begin{array}{l}\text { Stabilisation } \\
\text { Progression de la } \\
\text { référence visée } \\
\text { L'infraction aux } \\
\text { conventions } \\
\text { bloque }\end{array}$ & $\begin{array}{l}\text { Règlement du } \\
\text { conflit } \\
\text { Assurance de } \\
\text { changement social }\end{array}$ & $\begin{array}{l}\text { Conversion } \\
\text { Construction } \\
\text { bilatérale du sens } \\
\text { Valeur de vérité } \\
\text { d'une affirmation } \\
\text { Le désaccord ne } \\
\text { bloque pas }\end{array}$ \\
\hline
\end{tabular}


(la conversion du sujet y est attendue, dans le partage et la coélaboration de références) sont complétés par la discussion (faisant état de connivence, entre référence et cohérence thématique), ajoutée aux références fondatrices (Tableau I).

Des transactions entre ces strates (reprises et stratégies) permettraient de suivre des mouvements discursifs, en vue de relier les propos des interlocuteurs les uns aux autres, et permettraient d'approcher l'élaboration des significations.

\section{Méthodologie}

Rendre intelligible des produits et des processus au cours des échanges verbocognitifs scolaires n'induit pas une démarche d'investigation particulière. La prise en compte des sujets, l'instrumentation précisant les œuvres utilisées, le déroulement de l'étude, les méthodes d'analyse en corpus des résultats et le cadre éthique du recueil de données permettent de décrire la recherche en cours.

\subsection{Sujets}

Lors de séances de débats à propos de littérature en classe en fin de cycle primaire, des interactions cognitivo-langagières orales (environ 700 échanges) sont enregistrées dans trois classes (âge moyen: 10,6 ans environ), sur trois années consécutives. Ces classes, de la région de Bordeaux, aux profils ordinaires, mais hétérogènes, nous sont connues: des élèves avec lesquels nous avons travaillé l'année précédente (classe 1, classe témoin), ou dans lesquelles nous avons travaillé en tant qu'enseignante (classes 2 et 3, l'observation est participante). Les enregistrements sonores retracent le déroulement de la séance, et les notes prises après coup documentent le recueil de données.

Les situations d'enseignement recueillies mettent en ouvre des débats de lecture littéraire. Leur réalisation ne suit pas un cadre expérimental identique (les enseignants gardent leur liberté pédagogique). Des pratiques, ordinaires ou innovantes, sont soumises à l'étude.

1) Classe 1 (corpus 1): le travail de l'enseignant représente, pour lui, un débat interprétatif. Il porte sur la construction d'un horizon d'attente (premières hypothèses) d'un roman historique Le pionnier du nouveau monde (Piquemal, 1999). Les élèves doivent dire ce qui leur donne envie de lire ce livre ou pas, en décrivant la couverture. Des productions écrites sont réalisées à deux pendant 15 minutes. Elles sont présentées et débattues chacune leur tour durant 30 minutes, sur 150 échanges.

2) Classe 2 (corpus 2): dans la demi-classe 2, la dimension interprétative et compréhensive (Bakhtine, 1977) est sollicitée durant 60 minutes (prédominance du pourquoi de l'enseignant et des élèves, ou de que pensez-vous de...). Après la lecture d'un album Demain les fleurs (Lenain et Brouillard, 2001), une dyade d'élèves choisit une double page illustrée et la présente en s'appuyant sur le texte. Puis les élèves débattent de ce qu'ils ont compris de l'œuvre durant les 
dix dernières minutes. Ces 150 derniers échanges sont pris en compte, ainsi qu'une trace écrite de synthèse demandée a posteriori.

3) Classe 3 (corpus 3) : un débat est proposé à partir de la construction du point de vue de chacun des protagonistes de l'album Yakouba (Dedieu, 1984). Chaque groupe écrit le point de vue supposé d'un actant. Celui-ci est discuté puis confronté à celui d'un autre groupe pour le même protagoniste, le débat dure 40 minutes. Les 392 échanges des 40 minutes de ce travail sont étudiés, ainsi que les écrits concernant le point de vue des élèves, s'ils avaient été à la place de Yakouba.

\subsection{Instrumentation}

La diversité des situations recueillies ne permet pas d'envisager une dimension prescriptive au travail d'étude réalisée. Les albums choisis sont présentés au moyen d'une analyse a priori.

Les trois supports envisagés sont emblématiques des références culturelles utilisées fréquemment dans l'enseignement français, dont un résumé succinct et des caractéristiques, explicitent en partie nos choix effectués en tant que chercheuse.

- Le pionnier du Nouveau Monde (Piquemal, 1999) présente un jeune Français débarquant au Canada au XvII ${ }^{\mathrm{e}}$ siècle. À la suite d'une déception amoureuse, il découvre un univers inconnu à travers de multiples aventures surmontées par le courage (valeur morale) du héros, qui partage la dure existence des Indiens. Ce récit, prototype du roman historique, qui favorise les connaissances du monde (une terre inconnue, une confrontation culturelle), est doublé d'un roman d'amour et d'aventures (sollicitant des émotions fortes et valorisant les qualités humaines en relation avec les valeurs morales). Les deux enjeux sont importants: saisir comment les élèves s'emparent d'une telle complexité semble pertinent pour envisager la caractérisation d'un personnage.

- Demain les fleurs (Lenain et Brouillard, 2001) est un album qui raconte comment un enfant vit la fin d'un hiver qui n'en finit pas avec son grand-père. Aucun bourgeon sur l'arbre - qui a l'âge du grand-père - n'apparaït. Au village le plus proche, personne ne répond. Un soir, les deux personnages déchirent les pages d'un livre et déposent des fleurs en papier sur le pommier. C'est l'adieu du grand-père au garçon. Mais le lendemain, le grand-père est dans le jardin près du pommier en fleurs. Cet album, à la dimension poétique certaine, interroge la relation affective convoquant en arrière-plan les valeurs de vie et de mort dans des dimensions fortement symboliques, dont on peut se demander comment elle sollicite la construction des personnages pour de jeunes élèves.

- Yakouba (Dedieu, 1984) est un album qui retrace une étape de la vie d'un jeune Africain qui doit apporter la preuve de son courage pour montrer qu'il peut être un guerrier. Mais Yakouba se trouve devant un dilemme: tuer un lion blessé et passer pour un héros, ou laisser le lion vivre pour être en accord avec sa conscience, mais être mis à l'écart de sa tribu. Cet album représente le pro- 
totype du conte philosophique (les connaissances du monde sont davantage un arrière-plan), l'aventure est personnelle et intérieure, faisant appel à la conscience morale du héros, ce que les illustrations en noir et blanc renforcent. La complexité du dilemme nécessite de s’enquérir plus précisément de ce que les élèves comprennent de la situation, la sollicitation de l'élaboration d'un point de vue du personnage central semblant en adéquation avec la prise de conscience de ce dilemme.

Ces trois œuvres sont potentiellement porteuses de valeurs morales (à propos de différentes formes de courage) de façon plus ou moins intériorisée, et sollicitent a priori des verbalisations de sentiments, la dimension esthétique des œuvres semblant accessible a priori. Les écrits proposés en classe sollicitent la conscientisation des émotions sous la forme de sentiments par l'intermédiaire des dires que nous étudions.

\subsection{Déroulement}

L'approche de l'observation participante ou impliquée et l'approche à visée exploratoire contribuent à la possibilité de qualifier les échanges cognitivo-langagiers en permettant la reconstruction de significations intersubjectives possibles. Elles concourent à l'identification de manifestations de hiatus comme de potentielles ruptures cognitivo-verbales, en vue de mieux identifier les contenus d'apprentissage qui se construisent.

\subsubsection{Démarche exploratoire}

Une démarche d'investigation exploratoire est établie à partir d'un premier corpus recueilli et transcrit (le premier corpus restreint les données au verbatim pour être étudié, puis cette étude est étendue à tous les corpus). Les transcriptions tiennent compte des spécificités orales du corpus (Blanche-Benveniste, 2010) et ne sont ni retouchées ni éditées. Elles comportent des thématiques récurrentes, dont le rôle est à expliciter.

\subsubsection{Relations entre outils et contenus}

Une analyse des occurrences thématiques montre que les reprises ne se limitent pas à une répétition de termes stricto sensu. Qualifier l'écart entre les formulations amène à discerner la manière dont les propos sont formulés, et les rôles joués par ces propos en relation avec les contenus d'apprentissage deviennent un enjeu de recherche.

Ainsi, d'une part, les reprises-reformulations contribueraient à la construction d'une cohérence référentielle, mais aussi de contraintes thématiques plus ou moins bien assumées, et renseignent quant à la position des énonciateurs et des contenus disciplinaires sollicités. Par exemple, la caractérisation des personnages ou les intentions de l'auteur, la dimension symbolique, les valeurs sollicitées sont lisibles en filigrane. La construction de la notion de personnage, travaillée au cours des 
années de scolarisation précédentes, est encore délicate à identifier rapidement pour certains élèves. Cette caractérisation du personnage s'élabore à partir des reprises-reformulations qui ne permettent pas d'élucider immédiatement les emplois contradictoires du pronom il (Tableau I). Or l'accumulation de ces contradictions rend cette élucidation nécessaire.

D'autre part, les stratégies discursives permettraient de penser à la fois des contenus (d'apprentissage) et les modalités de réalisation des objets enseignés par le biais de la formulation de ces contenus, en vue de suivre la progression des discours dont la complémentarité est plus ou moins partagée, semblable à une négociation de l'intercompréhension, ou socioconstruction des significations.

\subsubsection{Conditions de recueil}

Les recueils d'informations, de données, collectées directement après l'oral ou plus tardivement, ont été réalisés par l'enseignante chercheuse. Ces informations sont soumises à des autorisations et à des masquages partiels développés ci-dessous.

\subsection{Méthodes d'analyse des données}

Les données sont observées à l'endroit des reprises-reformulations (François, 1989). Dans le cadre scolaire, elles semblent, selon nous, avoir des fonctions spécifiques. Ces reprises-modifications sont identifiées sous le nom de thématiques. Les niveaux de caractérisation des données transcrites, en relation avec les déplacements effectués par les modifications des reprises font entrevoir des lieux de tension. L'enjeu de l'étude envisage leur effet sur l'élaboration des connaissances, en particulier à l'occasion de ruptures de continuité thématique, ou de rupture de continuité de stratégie discursive. Des écarts nets entre des échanges consécutifs ou manifestations de hiatus sont établis, selon des critères discursifs (horizontalement ou verticalement, voir tableau II). Les négociations ou transactions des dires qui s'effectuent à cet endroit de hiatus seraient constitutives de traces de la construction d'un savoir comme contenu scolaire ou élaboration de problème.

\subsection{Considérations éthiques}

Le recueil d'informations a été effectué dans le cadre de travail d'une thèse. Les élèves et les maîtres participant à ces échanges ont autorisé la diffusion des données selon les modalités en vigueur en France auprès des institutions scolaires. Les noms et les prénoms sont rendus anonymes: les trois premières lettres des prénoms sont identiques, les origines culturelles des prénoms sont conservées. Le prénom d'un élève a été davantage masqué, à sa demande. Les élèves ont eu accès partiellement aux écoutes des enregistrements à titre individuel, dans chacune des classes. Les enseignants ont exprimé leurs impressions après chaque recueil. Les corpus des séances, transcrits, leur ont été transmis. La qualification et la quantification des critères retenus leur ont été fournies. Les constats auxquels 
ces analyses ont donné lieu leur ont été adressés afin de prendre en compte des remarques éventuelles de leur part. Des précisions ont été ajoutées. Aucune modification importante n'a eu à être apportée à la suite de ces échanges.

\section{Résultats}

La méthode d'analyse des données met l'accent sur la quasi-permanence de thématiques en relation et leur fluctuation dans les corpus. Ces reprises constituent jusqu'à un tiers des échanges. Trois exemples, caractéristiques des corpus de chaque classe, illustrent différents fonctionnements des reprises-modifications: présentes, elles sont plus ou moins représentatives d'apprentissages.

\subsection{Des reprises-modifications sans aboutissement apparent}

Dans la classe 1, les nombreuses reprises du terme Indien sont prouvées, 25 échanges plus loin, par la plume du personnage. Ce n'est pas une avancée discursive en soi, et cela interroge le contenu construit ou appris.

La forte fréquence des reprises produit un effet de spécification du champ lexical: les échanges à propos de la quête du nouveau monde laissent à penser que les élèves s'interrogent sur l'origine des personnages: des Indiens, dont la caractérisation reste implicite avec un autre homme, ou leur moyen de déplacement on dirait que c'étaient des canoës, de même que leur accommodation à des conditions de vie difficiles (se raser). Ces reprises convoquent des savoirs sur le monde (la présence de barbe ou pas selon l'origine ethnique). Elles tissent des relations ténues entre des thèmes évoqués et convoquent une expérience personnelle (un voyage au Québec pour l'élève qui a reconnu un canoë). Ces reprises sollicitent des inférences (mise en lien d'éléments de la couverture et connaissances sur le monde des élèves) sous la contrainte de la description. L'incitation des élèves à recourir à une explicitation de choix personnels est absente. L'argumentation est un prétexte, qui n'est qu'affiché dans la consigne: Pourquoi j'aime ou je n'aime pas ce livre, en décrivant la couverture. Les arguments cités pour dire que l'on aime différents aspects du roman proposés par les élèves évoquent un nouveau monde, le fait de connaitre l'aventure des personnages ou des préférences: aimer le canoë - le bateau, le sport -, établir la différence entre la peinture qui aurait pu servir à réaliser la couverture du roman et la couverture elle-même pour ce qu'elle permet de comprendre du roman à lire. Le point de vue inverse, je n'ai pas aimé parce qu'il n'y a pas beaucoup d'action, n'est ni repris ni interrogé dans le débat fortement dirigé par l'enseignant. Le hiatus majeur se situe entre les intentions contradictoires de l'adulte: d'une part, enrôler, impliquer les élèves dans la tâche en sollicitant leur appréciation et, d'autre part, convoquer un registre descriptif pour anticiper le récit, sans que de réels liens de cause à effet soient tissés entre ces deux entrées. D'autres hiatus renvoient à des parasitages (l'évocation du père Noël au moment des échanges à propos de la barbe contraint l'enseignant à reprendre les échanges pour rester dans le sujet). Si ces hiatus ne sont ni résolus ni exclus des 
échanges, ils entravent l'apparition de contenus de savoirs littéraires. Les propositions des élèves évoquent en pointillé des horizons d'attente qui ne sont pas confrontés au prologue lu. Les apprentissages possibles a priori (argumenter) ne sont pas concrétisés a posteriori.

\subsection{Des reprises-modifications et la construction référentielle des personnages}

Pour l'extrait du deuxième corpus de la classe 2, les reprises-formulations montrent une confusion dans l'utilisation des pronoms renvoyant aux personnages du récit: un enfant et son grand-père, ce qui est surprenant à la fin d'une si longue séance. Ces confusions (il, lui, le) reviennent à 14 reprises, dont huit fois dans les 25 premiers échanges des 10 dernières minutes. A priori, le contexte énonciatif est porteur d'enjeux liés aux valeurs reposant sur la vie et la mort, sur les émotions. En quoi ces reprises modifient-elles le propos de départ?

La confusion semble liée à une dimension occultée par la lecture de ces élèves: la relation unissant ces personnages. Ces élèves prennent conscience que cet enfant n'a plus que son grand-père et que celui-ci, comparé à un vieil arbre qui ne fleurit plus, peut mourir. Les reprises-modifications participent à la construction référentielle des personnages et de leurs relations: il est d'abord celui dont on parle (1) puis celui qui est modalisé ( 2 avec peut-être), avant que le doute ne s'installe dans la complexité à délimiter il et le. L'anticipation de scénario erroné (6) et une caractérisation du grand-père aboutissent à une hypothèse funeste. Un retour au flou de la désignation des personnages est pallié par la monstration (7), mais l'ambiguïté persiste, le hiatus est net (8). Des manifestations de hiatus reviennent au cours de l'avancée du discours, remettent en question la façon de dire cette avancée. Elles ont un rôle dans la construction d'une position métadiscursive, à partir de modalisations (échange 10 et suite: ... y a marqué l'enfant qui vit seul avec son grand-père, c'est pas dit que ses parents vont l'emmener..., si ça se trouve,... et c'est pas logique). A posteriori, l'étude permet de constater que les sentiments restent en arrière-plan, même s'ils irriguent la tension discursive des échanges (la mort est peu citée). Le courage ou la persistance du grand-père n'est pas évoqué, les intentions de l'auteur restent implicites. La construction référentielle du héros a été discutée et est explicite dans les traces écrites, la dimension symbolique est évoquée dans la moitié des résumés. Il semble que l'entrée d'un paramètre complexe (l'analogie entre l'arbre et la vie) nécessite la reconstruction des personnages et de leurs relations, les hiatus ou écarts entre ce qui est dit et compris participant à la construction de cette élaboration ardue, fortement négociée et traversée de ruptures. 
Tableau 2

Extrait du corpus 2 Demain les fleurs, à propos de l'ambiguité du pronom

\begin{tabular}{|c|c|c|}
\hline 1. Dia & $\begin{array}{l}\text { je ne comprends pas pourquoi } i l \text { dit } \\
\text { adieux au petit/pourquoi/il n'est pas } \\
\text { mort }\end{array}$ & $\begin{array}{l}\text { il: terme générique } \\
\text { Nég Cont } \\
\text { il: le même que } 1 \\
\text { Dial. Pr }\end{array}$ \\
\hline $\begin{array}{ll}\text { 2. Ant } \\
\text { 3. } M\end{array}$ & $\begin{array}{l}\text { peut-être qu'il ne le reverra pas } \\
\text { alors (?) }\end{array}$ & $\begin{array}{l}\text { il: reconfiguration/hypothèse } \\
\text { le: non précisé, l'autre } \\
\text { Négo. Fo } 1\end{array}$ \\
\hline 4. Ant & $\begin{array}{l}\text { peut-être qu'il croyait que c'était la } \\
\text { dernière fois qu'il le voyait }\end{array}$ & $\begin{array}{l}\text { Il: reprise d'un point de vue modalisé } \\
\text { Disc.form } \\
\text { il: sous forme d'hypothèse } \\
\text { le: non élucidé, l'autre } \\
\text { Dial. cont }\end{array}$ \\
\hline $\begin{array}{ll}\text { 5. } & M \\
\text { 6. } & \text { Emi }\end{array}$ & $\begin{array}{l}\text { tu écoutes (? adressé à Émile) } \\
\text { et que après il allait partir chez lui } \\
\text { encore avec ses parents et que/il serait } \\
\text { chez lui comme son grand-père était } \\
\text { très vieux et qu'il allait mourir sans/euh } \\
\text { voir dans ses derniers jours } \\
\text { son petit-fils (Elody retourne à la } \\
4^{e} \text { de couverture) }\end{array}$ & $\begin{array}{l}\text { II: le jeune garçon avec anticipation de scénario } \\
\text { implicite } \\
\text { lui: le même } \\
\text { reprise de il et de lui } \\
\text { il: caractérisation identificatoire } \\
\text { il: le grand-père qualifié induit une anticipation } \\
\text { de la mort, désambiguïsation avec tournure } \\
\text { complexe } \\
\text { Dial. cont }\end{array}$ \\
\hline 7. Ant & $\begin{array}{l}\text { et qu'à la dernière page il lui fait au } \\
\text { revoir il lui fait coucou (page montrée) }\end{array}$ & $\begin{array}{l}\text { Il: reprises descriptives situées et montrées du } \\
\text { doigt, } \\
\text { lui: reste implicite } \\
\text { Conv. fin }\end{array}$ \\
\hline 8. Sa & $\begin{array}{l}\text { et il lui dit au revoir et là ils se } \\
\text { promènent tous les deux/ça c'est pas } \\
\text { logique }\end{array}$ & $\begin{array}{l}\text { il: reprise à la suite de } 5 \text {, } \\
\text { ils pluriel précisé par tous les deux } \\
\text { Disc. cont }\end{array}$ \\
\hline 9. Ant & $\begin{array}{l}\text { oui mais ça il faut que tu continues/de } \\
\text { de }\end{array}$ & il: impersonnel Néq. dér \\
\hline 10. Ely & $\begin{array}{l}\text { maîtresse dans le résumé y a marqué/y } \\
\text { a marqué l'enfant est seul avec son } \\
\text { grand-père }\end{array}$ & $\begin{array}{l}\text { il: impersonnel } \\
\text { Ambiguitté partiellement levée sur le statut de } \\
\text { l'enfant Nég. dér }\end{array}$ \\
\hline
\end{tabular}

\subsection{Des reprises-modifications vers la problématisation}

Le troisième corpus de la troisième classe, découpé en thématiques plus nombreuses, est plus complexe à étudier. La construction d'un point de vue, éludée dans le travail écrit en groupe, prend sa pleine dimension au moment des échanges. Les nombreuses manifestations d'écarts ou de hiatus, que l'enseignante laisse apparaître ou qu'elle rappelle, interrogent les élèves. Ces ruptures contribuent à une problématisation des enjeux littéraires en utilisant les entrées de l'œuvre pour s'incarner en face du drame que doit assumer le héros: tuer pour la gloire ou s'assurer d'une conscience sereine. 


\section{Extrait $n^{0} 1$}

Extrait du corpus 1 : les échanges ne montrent aucune avancée discursive (Le pionnier du Nouveau Monde)

$60 \mathrm{M}$ : tu parles d'Indiens//d'accord/alors qui est-ce qui peut qui est-ce qui a vu des Indiens qui est-ce qui a marqué qui a des Indiens/oui vas-y Victor

$61 \mathrm{~V}$ : sur la première de couverture je vois

$62 \mathrm{M}$ : mais on entend rien Vic (Vic se lève)

$63 \mathrm{~V}$ : sur la première de couverture je vois un homme un Indien et un autre Indien et derrière/entre eux $y$ a des habits et des provisions (il lit ce qu'il $y$ a sur son cahier)

$64 \mathrm{X}$ : puis ils se promènent

$65 \mathrm{M}$ : écoutez bien/écoutez ce que dit euh Aïssa

66 Aï: on dirait que c'était des canoës

$67 \mathrm{~V}$ : ce sont des peaux de bêtes non

$68 \mathrm{xxx}$ : oui/ben/non/non c'est pas des Indiens

$69 \mathrm{M}$ : alors Indiens ou pas Indiens/ceux qui pensent que ce sont des Indiens lèvent le doigt (plus de doigts levés que tout à l'heure)/bien maintenant on va préciser est-ce que ce sont des Indiens pourquoi est-ce que vous pensez que ce sont des Indiens/alors on écoute Vic

Des scénarios imaginaires rendent possibles des actions fictives projetées par les élèves quant aux protagonistes du récit. Ces scénarios reconstruits sont renvoyés par l'enseignante et des élèves au texte: quelle validité ont-ils? Les points de vue élaborés par les élèves interrogent les valeurs respectives attribuées aux personnages. L'honneur ou le déshonneur du père, évoqué par le statut de Yakouba (échange 285: à la fin il est gardien de troupeau), le courage du héros (échange 286) ou la lâcheté de Yakouba, la gloire des guerriers (échange 285: il pourra pas devenir guerrier), la fierté ou la honte de Yakouba par rapport au bétail (idem) sont autant de thématiques mises en lien au cours de la présentation des différents points de vue des protagonistes évoqués. Les élèves s'appuient sur les sentiments prêtés aux personnages (échange 288 : il est encore accepté) et aux actants. Ils se réfèrent à leurs propres sentiments (échanges 289 ou 293 : quand tu es en colère, en - on - a envie), mais aussi aux sentiments des autres (échange 289 : si tu as déjà été en colère), à leurs expériences du réel (échange 289: tu dis tout ce qui te passe par la tête), à des expériences à venir (échange 293: quand t'envoie ton fils, on a envie qu'il y en ait beaucoup plus), au monde des récits (échange 291).

\subsection{Des scénarios fictionnalisés aux stratégies énonciatives}

L’avancée du discours contribue à l'élaboration de scénarios (échange 288) et à leur validité potentielle (Champagne-Vergez, 2010b), car la plupart des élèves avaient envisagé Yakouba victorieux du lion dans un premier temps.

Si les reprises-modifications sont caractéristiques de l'oral, elles n'assurent pas l'avancée didactique des contenus d'apprentissage visés, mais stabilisent des objets discursifs: ce sont des objets possibles et focalisés, dont la transaction reste à poursuivre ou à négocier. 
Le contenu scolaire correspondrait à un contenu thématique repris et modifié, identifié par les partenaires de l'échange, au déroulement établi, aux formes précisées et à des finalités perçues, dans un niveau discursif dit dialogué (échanges 285; en partie, 293).

\subsection{Les connaissances sollicitées}

Les connaissances littéraires - dans les extraits des corpus utilisés pour cet article - relèvent de la caractérisation des personnages ou plus précisément de leur construction en cours, à partir de différentes entrées:

- selon ce que les personnages font, à partir d'une explicitation indirecte à propos de la description de la couverture du roman historique Le pionnier du Nouveau Monde.

- selon les rapports que ces personnages entretiennent, pour deux corpus. Dans le premier corpus, la parentèle complexe qui unit le grand-père et le petit-fils (Demain lesfleurs) devient problématique avant d'être perçue. Dans le troisième corpus, des relations antagonistes participent du développement du point de vue de Yakouba par rapport à son père, au sein d'une société traditionnelle.

Ainsi assumer ses dires en tant qu'élève contribue-t-il à la construction d'une valeur identitaire et sociale, incontournable pour entrer dans une position de lecteur d'œuvres littéraires à l'école.

Ce qui se dit n'est pas seulement un contenu de connaissance ou de savoir, mais l'élaboration des conditions de la réalisation de ce contenu en tant qu'exemple issu ou étendu au champ littéraire, au cours de transactions consécutives, ce sur quoi nous revenons.

\section{Discussion des résultats}

Les analyses réalisées sur les trois corpus sont porteuses de pistes de réflexion quant aux outils utilisés et aux transactions cognitivo-langagières possibles à l'école primaire, par le biais d'un guidage du débat en particulier de la part du maître.

\subsection{Les reprises-modifications}

Des tissages de liens entre des thématiques par les reprises-modifications font envisager qu'elles entretiennent des relations aux contenus scolaires (le tiers des échanges étudiés, pour les trois corpus). Ces reprises construisent des usages du langage qui s'essaie à la langue et à ses modes de fonctionnement in situ, mesurant les effets de la langue (Bishop et Cadet, 2011). Les reprises-modifications, par les différents changements de statut des contenus discursifs qu'elles comportent, participent de la construction de savoirs littéraciés (Tauveron, 2002): les personnages, le contexte du récit, l'organisation axiologique des événements du récit à terme, à condition que les reprises soient tissées dans une relation qui ne se limite pas à de l'évocation (ce qui apparaît dans le corpus 1). 
Les variations de ces reprises-modifications et les négociations auxquelles elles donnent lieu restent des transactions à identifier. La caractérisation qualitative des reprises des corpus 1 et 2 amène à un constat: ces reprises sont insuffisantes pour établir une formulation définitionnelle à partir de laquelle tous les locuteurs s'entendent. Dans un corpus, la reprise thématique dérivée des personnages des Indiens masque l'objet d'apprentissage (un éventuel horizon d'attente construit), dès lors que les enjeux de sa résolution sont l'objet du discours du maître (corpus 1). Dans les deux autres corpus, la rupture potentielle, lorsqu'elle est visible, est porteuse d'une difficulté à dire, mobilisant des tensions (Nonnon, 2008) liées à la caractérisation délicate des personnages. Elle aboutit à la résolution partielle et ponctuelle de petits problèmes (corpus 2). Ces résolutions n'établissent pas un savoir stabilisé recontextualisable à propos d'autres œuvres à venir.

\subsection{Les stratégies énonciatives}

La complexité de formulation de savoirs négociés et dialogués (des contenus et les façons de les dire en relation étroite) pointe que ces savoirs appris sont contextualisés (les écrits font référence à des passages explicites: la colère). De nombreuses occasions sont nécessaires pour pouvoir aborder les savoirs littéraires de façon générale (le personnage). Cela nous interroge quant à ce qui est attendu comme formulation de savoirs littéraires à l'école primaire.

Les transactions négociées ou dialoguées, si elles sont superposables aux manifestations de hiatus, ou de rupture, sont plus fréquentes que celles identifiées a priori (corpus 3, un hiatus sur 17 échanges, pour un tous les 6 échanges en fin de recherche). Ces ruptures montrent des tensions d'ordres différents, sollicitant le langage comme instrument éthique, comme outil de travail sur soi et en relation avec les autres, dès lors que les valeurs humaines sont prises en charge par l'énonciateur élève (corpus 2, mais essentiellement dans le corpus 3).

\subsection{La technique sociale des sentiments}

Lors de l'expression des sentiments, la transaction rend possible l'entrée dans un interdiscours (Extrait $n^{\circ} 2$, corpus 3, échanges 285, 289 et 293), entre les mondes des locuteurs. Le travail des mots, dans les deux derniers corpus, prend en compte une intersignification à construire, même si la mort possible, comme angoisse incontournable, est peu aisément dicible (Tableau I, corpus 2, échange 6) dans la communauté où le discours devient public. Ce discours relie des mondes d'appartenance de l'élève, dans l'école par l'expression des dires et hors de l'école, par l'évocation des expériences personnelles à propos de la colère évoquée: je ne sais pas si tu as déjà été très très en colère (Extrait $\mathrm{n}^{\circ} 2$, corpus 3, échange 285).

Dans ce cas, on peut penser possible, comme le précise Rochex (2009, p. 28-30) que la littérature pourrait être l'un des moyens d'éviter le dessaisissement de soi (la disparition du sujet enfant dans l'élève) ou la dilution du soi dans l'institution scolaire. Le sujet (enfant et élève) ferait partie intégrante de la communauté dis- 


\section{Extrait $n^{0} 2$}

Extrait du corpus 3: un groupe d'élèves présente le point de vue du père lors d'échanges discursifs (Yakouba).

284 Fra Antime

285 Ant bien je suis d'accord avec toi si ils disent à la fin qu'il est gardien d'un troupeau il pourra pas devenir un guerrier/il le dit/en fait il est pas rejeté par sa famille mais les autres le considèrent pas comme un grand/quelqu'un qui peut faire des grandes choses on le

286 Fra il est pas assez reconnu

287 Ant voilà

288 Ken le papa (rires) sur ce papa/il va pas/puisqu'il est encore accepté il dort chez eux il va pas il va pas dire dégage

289 Fra le père je sais pas si tu as déjà été très très en colère mais quand tu es en colère tu dis tout ce qui te passe par la tête pour faire comprendre pour que comme parce que tu es très énervé après après

290 Ant là j'ai compris que vous vouliez carrément que Yakouba il s'en aille qu'il déménage

291 Nic $Y$ parce qu'au tout début on a mis ce qui pouvait euh les plus ceux qui avaient l'âge de combattre et Yakouba en était un

292 M dernière intervention de Duk

293 Duk moi pour le pire je suis d'accord et pas d'accord d'accord/parce que quand t'envoie ton fils en a envie que $y$ ait beaucoup plus de guerriers pour sauver un village et tout/on $(a)$ envie qu'il y en ait beaucoup plus et pas d'accord parce quand il a ce caractère-là c'est déjà qu'il reste plus ici même s'il voulait que les meilleurs restent et que les autres///

$294 M$ bon là on a

cursive scolaire. La relation entre la subjectivation (ce que l'élève ressent), ses sentiments et un langage personnel (ce que pense ou ce que dit chacun) peut devenir un des enjeux essentiels de la littérature.

\subsection{Vers une dialogisation}

Le langage permet de penser l'intercompréhension entre les sujets (ce qu'ils en pensent et ce qu'ils en disent), dans un dialogue possible. De même, Jacques (2007) et Gardin (2008), entre autres, font référence à l'élaboration d'un sujet par la construction d'un sens éthique et par l'élaboration d'un discours dialogique, au sens du partage des enjeux des échanges et des contenus abordés.

La focalisation sur le travail des mots effectué par les élèves rend visible un levier plus ou moins efficient.

\subsection{Les gestes professionnels et le travail de transaction des mots}

Le travail de transaction, en relation à la technique des sentiments et en phase avec les sollicitations de l'enseignant, permet de mieux comprendre comment le langage parle aux élèves. Le langage dit à chacun et à tous, en même temps qu'il construit le dialogue au sens dialogique (Bakhtine et Volochinov, 1977; Jacques, 1988). Il permet aux uns et aux autres de comprendre et de se comprendre (intercompréhension). En effet, aimer ou ne pas aimer, renvoyer aux mythes fondateurs 
ou aux questions fondamentales pour l'homme, à la présence ou à l'absence - de l'affection portée aux parents, aux proches -, aux valeurs de courage ou par défaut de lâcheté, à la conscience tranquille, convoquent le sujet lecteur dans son intimité. À cette occasion, une conscientisation des affects prend forme, par la médiation de l'œuvre, mais aussi par la médiation de la dimension sociale de la convocation de l'œuvre, en particulier à l'école primaire.

L'élève, considéré en tant que sujet autonome en devenir, apprend à communiquer par, avec et dans la parole. Cette parole est le lieu d'un travail, entre travail personnel - intériorisé et dont seules des traces restent visibles - et travail public, au sein de communautés d'appartenances, en vue de négocier des significations complexes, ponctuelles et en vue de les généraliser, ce dont le maître est garant.

\subsection{Le hiatus}

Une focalisation à l'endroit d'éventuels malentendus, liés à la polysémie ou à la superposition des registres approximatifs des significations en construction, pourrait favoriser l'identification de points nodaux de discours à négocier.

\subsection{Limites}

Une telle étude n'est pas sans soulever un certain nombre de questions qui sont autant de limites potentielles:

- Bien que le nombre d'échanges étudiés soit relativement important, les situations restent singulières, dépendantes à la fois des intervenants, des supports, des choix, des enjeux d'enseignement identifiés et identifiables, au-delà de la compétence sociale et civique potentielle.

- La recherche d'un marqueur linguistique (le hiatus) est davantage un révélateur pour le chercheur qui rend lisible l'élaboration des interactions constitutives des contenus à apprendre qu'un éventuel outil exploitable dans la conduite de la classe, étant donné sa dimension peu apparente a priori.

- Cette étude montre la complexité des niveaux d'observation à mobiliser en vue de comprendre ce que les échanges langagiers sollicitent en termes de connaissances et de compétences, au-delà des contenus ordinairement pensés à acquérir.

- Dans cet article, nous avons envisagé essentiellement des dimensions orales, il est cependant important de rappeler le rôle de lecteur-scripteur qui amplifie les enjeux discursifs liés à la littérature.

- C'est éventuellement sur cette nécessité de négocier et de dialoguer que l'enseignant peut intervenir, sollicitant des ajustements des locuteurs en présence (Bucheton, 2011), dans la mesure où les locuteurs acceptent ce processus de négociation et de dialogue (afin de se déprendre d'un pouvoir discursif enseignant souvent unilatéral). Ainsi la mise à disposition des élèves de situations riches relève des gestes professionnels anticipant les apprentissages, ainsi que d'un effacement en vue de dévoluer la lente construction du problème littéraire aux élèves. 
- Les propositions relatées sont ponctuelles et nécessiteraient une mise en œuvre expérimentale plus élaborée en vue de confirmer les avancées constatées.

Une mise en perspective des hypothèses envisagées ponctue cette réflexion.

\section{Conclusion}

L'hypothèse de départ se focalise sur le conflit potentiel lié aux tensions, dues à des manifestations de ruptures, d'incohérences, de hiatus, qui participent à la construction d'un problème, propice à l'élaboration de significations pour le sujet lecteur interprète. Ce problème était centré sur la construction identitaire des personnages. Celle-ci s'élaborerait à partir de la sollicitation de reprises variées participant à un dire socioconstruit à partir de valeurs, d'émotion esthétique, d'une technique sociale des sentiments, en parallèle avec les gestes professionnels des enseignants et avec l'instauration d'une communauté discursive.

La perspective de l'investigation de départ est multiple: elle recherche des indices de valeurs (esthétiques, morales et humaines) engagées dès l'école primaire, par le biais d'une médiation culturelle privilégiée : la technique sociale des sentiments. L'étude prend en compte des hypothèses avérées en partie seulement, en s'appuyant sur des outils plus ou moins adéquats (l'identification des stratégies énonciatives est complexe) et selon une technique des sentiments dont les usages restent à cerner, à l'école primaire du moins. Cette entrée, peu visible sans les outils d'investigation utilisés, est possible, sans être systématique. Ce travail s'effectue lorsque les registres thématiques sollicités par les élèves interrogent des liens qui unissent ces thèmes selon leurs questions, en dehors d'un guidage étroit de l'enseignant. L'effacement de l'enseignant en serait-il une garantie relative? Il est probablement rendu possible par le fonctionnement d'une communauté discursive disciplinaire, ici littéraire, aux usages installés et en devenir.

La méthode proposée cible des manifestations de hiatus potentiellement omniprésents. Les outils méthodologiques utilisés ne sont pas tous aussi pertinents pour les identifier. La reprise-modification permet de focaliser des objets du discours en cours d'élaboration, elle participe à la stabilisation des contenus et met en exergue les effets de la langue. Les reprises-modifications et leurs conditions d'émergence délimitent des contenus notionnels pour un tiers des échanges. Elles participent aux constructions des problématiques à la fois littéraires (le personnage, sa situation, des situations possibles, leurs devenirs) et humanistes (les espoirs, la dimension symbolique, les valeurs probables rencontrées par les personnages). Les outils de la recherche deviennent des outils de guidage de l'action qui restent à finaliser. Les stratégies énonciatives montrent des écarts importants entre des registres définitionnels sollicités (Tableau I). Des tensions surgissent, dès lors que la continuité référentielle n'est pas partagée entre des échanges consécutifs (manifestation de hiatus). Ainsi des transactions sociales portent à la fois sur des contenus d'enseignements et sur des modalités discursives du domaine littéraire (deux tiers des échanges), étudiées en tant que stratégies conversationnelles. Ces transactions font 
constater la complexité des échanges langagiers et des opérations cognitives qui se déroulent au cours du débat, sous une forme de comment dire en contexte.

La technique sociale des sentiments est plus ou moins sollicitée. Les résultats font état de la dimension d'un sujet lecteur exprimant ses sentiments de façon implicite dans deux corpus sur trois. Cependant capable d'envisager l'argumentation de ses choix avec ses pairs, chaque sujet élève singulier peut faire part de ses sentiments comme d'une expérience intérieure, subjective et sociale plus objectivée. Chacun utilise les tensions entre la façon de dire et le contenu ou la forme et le matériau de l'œuvre (selon Vygotski, 2005) pour construire ses représentations littéraires, ancrées dans un ressenti et un vécu, par appropriation. Le fait langagier médiatise la transaction de significations à propos des sentiments et des valeurs supposées pour autrui. Ce travail est rendu possible par l'activité proposée, au sein d'une situation adéquate, en particulier lors de l'élaboration d'un point de vue à faire partager aux autres. Les valeurs sont discutées et mises à l'épreuve virtuellement, devenant l'objet d'un travail au sens d'une socioconstruction des significations.

Le rôle de l'enseignant est important: ses actions langagières donnent lieu à des transactions, qu'il lui appartient de guider sans les diriger (le risque de masquage des enjeux accessibles est réel, corpus 1), utilisant un registre éthique de l'outil langagier. Si la circulation de la parole est un préalable, elle ne suffit pas à construire des connaissances. De plus, il reste à penser les élèves capables de se construire en sujets éthiques avec, dans et par le langage. La pratique langagière en situation contribuerait à une meilleure maîtrise de la langue à long terme. En effet, dans ces situations discursives, les élèves s'essayent à des genres, sans que soit visée une maîtrise formelle figée et normée, pour installer une connaissance progressive de la langue.

Le choix des œuvres proposées n'est pas neutre. Si l'on pense primordial de sensibiliser à une production locale et variée, ce choix doit aussi se porter sur des enjeux éthiques très complexes à percevoir pour les élèves. Bien que la construction du personnage en littérature soit préconisée dès la maternelle à travers l'introduction de récits dans les instructions officielles françaises, et que les questions sur les personnages soient omniprésentes pour les enseignants dans les classes, la dimension éthique et les enjeux de ces sollicitations sont souvent minorés. L’exemple de cet article, centré sur le personnage, précise la complexité de cette notion, non seulement quant à son identification, mais aussi à son rôle, ce qui nécessite un guidage pour renvoyer les élèves aux valeurs et à leurs effets.

Des prolongements de cette recherche à d'autres niveaux de l'école primaire sont en cours d'étude. L'intérêt de questionner un enseignement et un apprentissage curriculaire renvoie à la conception des objets disciplinaires à enseigner. Les objets d'enseignement ne se limitent pas aux notions, ils interrogent les processus pour y parvenir, comme moyens de piloter l'activité en littérature, et pour favoriser le développement de l'enfant et des valeurs humaines. En garantissant une 
offre de médiation, l'enseignant accorde une qualité aux échanges cognitivolangagiers - dont la preuve est délicate à établir, il saisit plus ou moins rapidement les enjeux éthiques de ces échanges. L'enseignant deviendrait passeur culturel dès lors qu'il permettrait un tissage de liens entre les différentes instances possibles du sujet lecteur et l'œuvre, notamment en prenant appui sur l'identification des constructions possibles du personnage en fin d'école primaire.

ENGLISH TITLE - Sentiments related to literary knowledge: transaction of words and values construction at elementary school and corpus analyses

SUMMARY - Teachers use words to mediate between literary works and their pupils. How the expression of aesthetic emotion contributes to build literary knowledge? What is the relation between sentiments and the construction of values? This study is based upon a corpus of three oral and written productions collected at the end of primary school in France (7th grade). In this article, we focus on the discursive repeats which emerge from the interpretative debate. The building of meaning, elaborated by the teacher and the pupils in a cooperative process, allows us to examine the building of values and knowledge.

KEY WORDS - language actions, school literary practices, interpretation, building of knowledge, values.

TítuLo - De los sentimientos a los conocimientos literarios: trabajo de "transacción" de palabras, construcción de valores en la primaria y análisis de corpus

RESUMEN - El docente, como facilitador privilegiado del acceso a las obras literarias, inicia al respecto una mediación a través del trabajo con las palabras, particularmente de índole estética y emocional. ¿Cómo participa la expresión de la emoción estética en lo que se aprende durante las actividades literarias escolares? ¿Cuál es la relación entre los sentimientos y la construcción de valores? Nuestro estudio se basa en tres corpus orales y escritos, colectados al final de la primaria en Francia ( $5^{\circ}$ grado). La observación de las acciones lingüísticas se sitúa a nivel de las reproducciones discursivas del debate interpretativo. En algunos casos, transacciones elaboradas conjuntamente por alumnos y docente, tanto al oral como al escrito, llevan rastros de esta elaboración de los saberes y valores.

PALABRAS CLAVE - acciones lingüísticas, actividades literarias escolares, debate interpretativo, elaboración de saberes, valores. 


\section{Références}

Ahrs, S. (2007). Pour une généricité lectoriale dans le secondaire. Construire et exploiter des corpus de genre scolaires. Enjeux, 10, 75-88.

Bakhtine, M. (1984). Esthétique de la création verbale. Paris, France: Presses universitaires de France.

Bakhtine, M. et Volochinov, N.-V. (1977). Le marxisme et la philosophie du langage. Essai d'application de la méthode sociologique en linguistique. Paris, France: Presses universitaires de France.

Barré de Miniac, C. (2008). Le rapport à l'écriture: une valeur heuristique. Diptyque, 12, 11-23.

Bernié, J.-P. (2002). L'approche des pratiques langagières scolaires à travers la notion de "communauté discursive» : un apport à la didactique comparée? Revue française de pédagogie, 141, 77-88.

Bernié, J.-P. et Goigoux, R. (dir.) (2005). Quels concepts pour quelle analyse de l'activité des enseignants de français? Lettre de l'Association Internationale pour la Recherche en Didactique du Français, 36(1), 3-4.

Bernié, J.-P., Jaubert, M. et Rebière, M. (2003). L'hypothèse d'une communauté discursive: d'où vient-elle? Où va-t-elle? Cahiers Théodile, 4, 51-80.

Bishop, M.-F. et Cadet, L. (2011). Maîtrise de la langue et compétence langagière. Le français aujourd'hui, 173, 3-8.

Blanche-Benveniste, C. (2010). Approches de la langue parlée en français. Paris, France: Ophrys.

Boiron, V. (2008). Former de jeunes lecteurs d'albums à l'école primaire. Modernités, 28, 277-290.

Bronckart, J.-P. (1997). L'activité langagière. Textes et discours. Pour un interactionnisme socio-discursif. Neufchâtel, Suisse / Paris, France: Delachaux et Niestlé.

Bucheton, D. (dir.) (2011). L’agir enseignant: des gestes professionnels ajustés. Toulouse, France: Octarès Éditions.

Burgos, M. (1996). Les débats de lecture: pratiques d'appropriation ou construction dialogique des textes? Dans J.-L. Dufays, L. Gemenne et D. Ledur (dir.), Pour une lecture littéraire, tome 2 (p. 265-271). Bruxelles, Belgique: De Boeck.

Butlen, M. et Dubois-Marcoin, D. (2005). Présentation. La littérature de jeunesse: repères, enjeux et pratiques. Le français aujourd'hui, 149, 3-6.

Champagne-Vergez, M. (2010a). Phénomène et manifestations de hiatus au sein des interactions langagières orales: des révélateurs d'obstacles aux apprentissages scolaires en fin d'école primaire (Thèse de doctorat non publiée). Université de Bordeaux 2, Récupéré du site: http://www.theses.fr/2010BOR21714/abes

Champagne-Vergez, M. (2010b). La question des valeurs éthiques dans les interactions langagières élaborées à propos d'un texte littéraire. Dans B. Louichon et A. Rouxel (dir.), La littérature en corpus. Bourgogne, France: SCÉRÉN (Services Culture Édition Ressources pour l'Éducation nationale).

Charaudeau, P. et Maingueneau, D. (2002). Dictionnaire d'analyse du discours. Paris, France: Seuil. 
Crahay, M. (2010). Qualitatif, quantitatif: des enjeux convergents? Dans L. Paquay, M. Crahay et J.-M. De Ketele (dir.), L'analyse qualitative en éducation. Des pratiques de recherche aux critères de qualité. Hommage à M. Huberman (p. 35-55). Bruxelles, Belgique: De Boeck.

Damasio, A. (2003). Spinoza avait raison: joie et tristesse, le cerveau des émotions. Paris, France: Odile Jacob.

Daunay, B. (2007). Le sujet lecteur: une question pour la didactique du français. Le français aujourd'hui, 137, 43-51.

* Dedieu, T. (1984). Yakouba. Paris, France: Seuil.

Dias-Chiaruttini, A. (2011). Émergence du débat interprétatif dans l'enseignement de la lecture/littérature à l'école primaire française. Lettre de l'AIRDF, 49, 10-14.

Dubois Marcoin, D. et Tauveron, C. (dir.) (2008). Éditorial. Pratiques effectives de la littérature à l'école et au collège. Repères, 37, 3-26.

Dufays, J.-L. (2004). La dialectique des valeurs. Le jeu très ordinaire de l'évaluation littéraire. Diptyque, 2, 103-129.

Dumortier, J.-L. (2001). Lire le récit de fiction. Pour étayer un apprentissage: théorie et pratique. Bruxelles, Belgique: De Boeck et Larcier.

Gardin, B. (2008). Pour une étude des morales langagières. Dans R. Delamotte-Legrand et C. Caïtucoli (dir.), Morales langagières. Hommage à B. Gardin (p. 19-26). MontSaint-Aignan, France: Presses universitaires de Rouen et du Havre.

Gion, M.-L. (dir.) (2003). Les chemins de la littérature au cycle 3. Créteil, France: Centre régional de documentation pédagogique de l'académie de Créteil, SCÉRÉN (Services Culture Édition Ressources pour l'Éducation nationale).

Hébert, M. (2002). Co-élaboration du sens dans les cercles littéraires entre pairs en première secondaire: étude entre les modèles de lecture et de collaboration (Thèse de doctorat non publiée). Université de Montréal, Montréal. Récupéré du site: http://theses.umontreal.ca/theses/nouv/hebert_m/theses.pdf

François, F. (1989). Langage et pensée: dialogue et mouvement discursif chez Vygotski et Bakhtine. Enfance, 42(1-2), 39-48.

François, F. (2005). Interprétation et dialogue chez des enfants et quelques autres. Lyon, France: ENS Éditions.

Jacques, F. (1979). Dialogiques. Recherches logiques sur le dialogue. Paris, France: Presses universitaires de France.

Jacques, F. (1988). Trois stratégies interactionnelles: conversation, négociation, dialogue. Dans J. Cosnier et C. Kerbrat-Orecchioni (dir.), Échanges sur la conversation. Paris, France: Centre national de la recherche scientifique.

Jacques, F. (2007). Éthique et langage. Dans M. Castilllo, F. Jacques, M. Humeau et F. Marty (dir.), L'éthique du rapport au langage. Paris, France: L'Harmattan.

Jorro, A. (1999). Le lecteur interprète. Paris, France: Presse universitaires de France.

Jorro, A. et Crocé-Spinelli, H. (2010). Le développement de gestes professionnels en classe de français. Le cas de situation de lecture interprétative. Pratiques, 145/146, 125-140.

Lahire, B. (1993). La raison des plus faibles. Rapport au savoir, écritures domestiques et 
lecture des milieux populaires. Lille, France: Presses universitaires de Lille.

Langlade, G. et Rouxel, A. (dir.) (2004). Le sujet lecteur. Lecture subjective et enseignement de la littérature. Rennes, France: Presses universitaires de Rennes.

Leclaire-Halté, A. (2006). Lecture formelle ou participative? Première réception d'Histoire à quatre voix au cycle 3 . Lidil, 33, 117-134.

Lelièvre, C. (2009). Culture humaniste et «socle» de l'école obligatoire (tout le reste est littérature). Le français aujourd'hui, 167, 11-18.

* Lenain, T. et Brouillard, A. (2001). Demain les fleurs. Paris, France: Édition Magnard.

Martinand, J.-L. (2000). Pratiques de référence. Dans A. Terrisse (dir.), Didactique des disciplines: la référence au savoir (p. 17-24). Bruxelles, Belgique: De Boeck.

Nonnon, E. (2008). Travail des mots, travail de la culture et migration des émotions: les activités de français comme techniques sociales du sentiment. Dans M. Brossard et J. Fijalkov (dir.), Vygotski et les recherches en éducation et en didactiques (p. 91-121). Pessac, France: Presses universitaires de Bordeaux.

* Piquemal, M. (1999). Le pionnier du Nouveau Monde. Toulouse, France: Milan poche jeunesse.

Reuter, Y. (1998). L'importance du personnage. Pratiques, 60, 3-22.

Ricœur, P. (1984). La configuration dans le récit de fiction. Paris, France: Seuil.

Rochex, J.-Y. (2009). Expérience scolaire et procès de subjectivation. Le français aujourd'hui, 166, 21-32.

Rosenblatt, L. (1994). The reader, the text, the poem, the transactional theory of literacy work. Carbondale / Southern Illinois University Press.

Rosenblatt, L. (1995:1938). Litterature as exploration. New York, New York: The modern language association of America ( $5^{\text {th }}$ édition).

Rosier, J.-M. (2010). Le principe de la dévolution dans le dispositif de formation/enseignement de la culture commune en Belgique francophone. Enjeux, 78, 17-32.

Tauveron, C. (1999). Comprendre et interpréter le littéraire à l'école: du texte réticent au texte proliférant. Repères, 19, 9-38.

Tauveron, C. (dir.) (2002). Lire la littérature à l'école. Pourquoi et comment conduire cet apprentissage spécifique. De la GS au CM. Paris, France: Hatier.

Vygotski, L.-S. (1998). Théorie des émotions. Étude historico-psychologique. Paris, France: L'Harmattan.

Vygostki, L.-S. (2003). Conscience, inconscient et émotions. Paris, France: Éditions La Dispute.

Vygostski, L.-S. (2005). Psychologie de l’art. Paris, France: Éditions La Dispute.

Madame Champagne-Vergez est membre de l'équipe E3D (Enseignement des didactiques disciplinaires) du LACES de Bordeaux 2 et membre du réseau Réseida (Escol Paris VIII).

\section{Correspondance}

champagnevergez@orange.fr 


\section{Contribution de l'auteure}

Martine Champagne-Vergez: $100 \%$

Ce texte a été révisé par Nancy Allen.

Texte reçu le: 5 juin 2011

Version finale reçue le: 17 juillet 2013

Accepté le: 15 août 2013

\section{Annexe 1}

Le socle commun des connaissances et des compétences, cadre de référence de la scolarité obligatoire (décret 2006-830-30 du 11 juillet 2006), s'appuie sur sept compétences:

1- la maîtrise de la langue française;

2- la pratique d'une langue vivante étrangère;

3- les principaux éléments de mathématiques et la culture scientifique et technologique;

4- la maîtrise des techniques usuelles de l'information et de la communication;

5- la culture humaniste;

6- les compétences sociales et civiques;

7- l'autonomie et l'esprit d'initiative. 